\title{
A LINEAR LEAST SQUARES FIT MAPPING METHOD FOR INFORMATION RETRIEVAL FROM NATURAL LANGUAGE TEXTS
}

\author{
YIMING YANG \\ CHRISTOPHER G. CHUTE
}

\author{
Section of Medical Information Resources \\ Mayo Clinic/Foundation \\ Rochester, Minnesota 55905 USA
}

\begin{abstract}
This paper describes a unique method for mapping natural language texts to canonical terms that identify the contents of the texts. This method learns empirical associations between free-form texts and canonical terms from human-assigned matches and determines a Linear Least Squares Fit (LLSF) mapping function which represents weighted connections between words in the texts and the canonical terms. The mapping function enables us to project an arbitrary text to the canonical term space where the "transformed" text is compared with the terms, and similarity scores are obtained which quantify the relevance between the the text and the terms. This approach has superior power to discover synonyms or related terms and to preserve the context sensitivity of the mapping. We achieved a rate of $84 \%$ in both the recall and the precision with a testing set of 6,913 texts, outperforming other techniques including string matching (15\%), morphological parsing $(17 \%)$ and statistical weighting ( $21 \%)$.
\end{abstract}

\section{Introduction}

A common need in natural language information re trieval is to identify the information in free-form texts using a selected set of canonical terms, so that the texts can be retrieved by conventional database techniques using these terms as keywords. In medical classification, for example, original diagnoses written by physicians in patient records need to be classified into canonical disease categories which are specified for the purposes of research, quality improvement, or billing. We will use medical examples for discussion although our method is not limited to medical applications.

String matching is a straightforward solution to automatic mapping from texts to canonical terms. Here we use "term" to mean canonical description of a concept, which is often a noun phrase. Given a text (a "query") and a tet of canonical terms, string matching counts the common words or phrases in the text and the terms, and chooses the term containing the largest overlap as most relevant. Although it is a simple and therefore widely used technique, a poor success rate (typically 15\%-20\%) is observed [1]. String-matchingbased methods suffer from the problems known as "too little" and "too many". As an example of the former, high blood pressure and hypertension are synonyms but a straightforward string matching cannot capture the equivalence in meaning because there is no common word in these two expressions. On the other hand, there are many terms which do share some words with the query high blood pressure, such as high head at term, fetal blood loss, etc.; these terms would be found by a string matcher although they are conceptually distant from the query.

Human-defined synonyms or terminology thesauri have been tried as a semantic solution for the "too little" problem [2] [3]. It may significantly improve the mapping if the right set of aynonyms or thesaurus is available. However, as Salton pointed out [4], there is "no guarantee that a thesaurus tailored to a particular text collection can be usefully adapted to another collection. As a result, it has not been possible to obtain reliable improvements in retrieval effectiveness by using thesauruses with a variety of different document collections".

Salton has addressed the problem from a different angle, using statistics of word frequencies in a corpus to estimate word importance and reduce the "too many" irrelevant terms [5]. The idea is that "meaningful" words should count more in the mapping while unimportant words should count less. Although word counting is technically simple and this idea is commonly used in existing information retrieval systems, it inherits the basic weakness of surface string matching. That is, words used in queries but not occurring in the term collection have no affect on the mapping, even if they are synonyms of important concepts in the term collection. Besides, these word weights are determined regardless of the contexts where words have been used, so the lack of sensitivity to contexts is another weakness.

We focus our efforts on an algorithmic solution for achieving the functionality of terminology thesauri and semantic weights without requiring human effort in identifying synonyms. We to capture such knowledge through samples representing its usage in various contexts, e.g. diagnosis texts with expert-assigned canonical terms collected from the Mayo Clinic patient record archive. We propose numerical method, a "Linear 
(a) text/erm pairs and the matrix representation

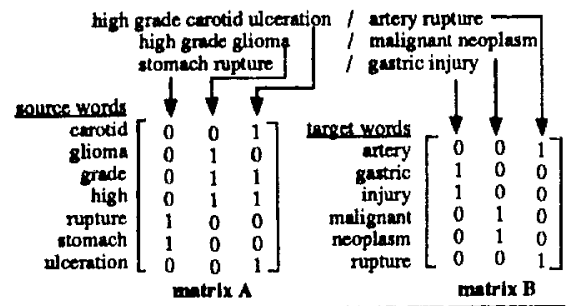

(b) an LLSF solution W of the linear system WA $=\mathrm{B}$

carotid glioma grade high rupture stomach ulceration

martery
malignicy $\left[\begin{array}{ccccccc}0.375 & -0.25 & 0.125 & 0.125 & 0 & 0 & 0.375 \\ 0 & 0 & 0 & 0 & 0.5 & 0.5 & 0 \\ 0 & 0 & 0 & 0 & 0.5 & 0.5 & 0 \\ -0.25 & 0.5 & 0.25 & 0.25 & 0 & 0 & -0.25 \\ -0.25 & 0.5 & 0.25 & 0.25 & 0 & 0 & -0.25 \\ 0.375 & -0.25 & 0.125 & 0.125 & 0 & 0 & 0.375\end{array}\right]$

Figure 1. The matrix representation of a text/term pair collection and the mapping function $W$ computed from the collection.

Least Squares Fit" mapping model, which enables us to obtain mapping functions based on the large collection of known matches and then use these functions to determine the relevant canonical terms for an arbitrary text.

\section{Computing an LLSF mapping function}

We consider a mapping between two languages, i.e. from a set of texts to a set of canonical terms. We call the former the source language and the latter the target language. For convenience we refer to an item in the source language (a diagnosis) as "text", and an item in the target language (a canonical description of a disease category) as "canonical term" or "term". We use "text" or "term" in a loose sense, in that it may be a paragraph, a sentence, one or more phrases, or simply a word. Since we do not restrict the syntax, there is no difference between a text and a term, both of them are treated as a set of words.

\subsection{A numerical representation of texts}

In mathematics, there are well-established numerical methods to approximate unknown functions using known data. Applying this idea to our text-to-term mapping, the known data are text/term pairs and the unknown function we want to determine is a correct (or nearly correct) text-to-term mapping for not only the texts included in the given pairs, but also for the texts which are not included. We need a numerical representation for such a computation.

Vectors and matrices have been used for representing natural language texts in information retrieval systems for decades [5]. We employ such a representation in our model as shown in Figure 1 (a). Matrix $A$ is a set of texts, matrix $B$ is a set of terms, esch column in $A$ represents an individual text and the corresponding column of $B$ represents the matched term. Rows in these matrices correspond to words and cells contain the numbers of times words occur in corresponding texts or terms.

\subsection{The mapping function}

Having matrix $A$ and $B$, we are ready to compute the mapping function by solving the equation $W A=B$ where $W$ is the unknown function. The solution $W$, if it exists, should satisfy all the given text/term pairs, i.e. the equation $W \vec{a}_{i}=\vec{b}_{i}$ holds for $i=1, \ldots, k$, where $\boldsymbol{k}$ is the number of text/term pairs, $\vec{a}_{i}(n \times 1)$ is a text vector, a column of $A ; \vec{b}_{i}(m \times 1)$ is a term vector, the corresponding column in $B ; n$ is the number of distinct source words and $m$ is the number of distinct target words.

Solving $W A=B$ can be straightforward using techniques of solving linear equations if the system is consistent. Unfortunately the linear system $W A=B$ does not always have a solution because there are only $m \times n$ unknowns in $W$, but the number of given vector pairs may be arbitrarily large and form an inconsistent system. The problem therefore needs to be modified as a Linear Least Squares Fit which always has at least one solution.

Definition 1 . The LLSF problem is to find $W$ which minimizes the sum

$$
\sum_{i=1}^{k}\left\|\vec{e}_{i}\right\|_{2}^{2}=\sum_{i=1}^{k}\left\|W \vec{a}_{i}-\vec{b}_{i}\right\|_{2}^{2}=\|W A-B\|_{F}^{2},
$$

where $\vec{e}_{i} \stackrel{\text { def }}{=} W \vec{a}_{i}-\vec{b}_{i}$ is the mapping error of the $i$ th text/term pair; the notation $\|\ldots\|_{2}$ is vector 2-norm, defined as $\|\vec{v}\|_{2}=\sqrt{\sum_{j=1}^{m} v_{j}^{2}}$ and $\vec{v}$ is $m \times 1 ;\|\ldots\|_{F}$ is the Frobenius matrix norm, defined as

$$
\|M\|_{F}=\sqrt{\sum_{i=1}^{k} \sum_{j=1}^{m} m_{i j}^{2}}
$$

and $M$ is $m \times k$.

The meaning of the LLSF problem is to find the mapping function $W$ that minimizes the total mapping errors for a given text/term pair collection (the "training 
set"). The underlying semantics of the transformation $W \vec{a}_{i}=\vec{b}_{i}$ is to "translate" the meaning of each source word in the text into a set of target words with weights, and then linearly combine the translations of individual words to obtain the translation of the whole text. Figure 1 (b) is the $W$ obtained from matrix $A$ and $B$ in (a). The columns of $W$ correspond to source words, the rows correspond to target words, and the cells are the weights of word-to-word connections between the two languages. A little algebra will show that vector $\vec{b}_{i}=W \vec{a}_{i}$ is the sum of the column vectors in $W$, which correspond to the source words in the text.

The weights in $W$ are optimally determined according to the training set. Note that the weights do not depend on the literal meanings of words. For example, the source word glioma has positive connections of 0.5 to both the target words malignant and neoplasm, showing that these different words are related to a certain degree. On the other hand, rupture is a word shared by both the source language and the target language, but the source word rupture and the target word rupture have a connection weight of 0 because the two words do not co-occur in any of the text/term pairs in the training set. Negative weight is also possible for words that do not co-occur and its function is to preserve the context sensitivity of the mapping. For example, high grade in the context of high grade carotid ulceration does not lead to a match with malignant neoplasm, as it would if it were used in the context high grade glioma, because this ambiguity is cancelled by the negative weights. Readers can easily verify this by adding the corresponding column vectors of $W$ for these two different contexts.

\subsection{The computation}

A conventional method for solving the LLSF is to use singular value decomposition (SVD) [6] [7]. Since mathematics is not the focus of this paper, we simply outline the computation without proof.

Given matrix $A(n \times k)$ and $B(m \times k)$, the computation of an LLSF for $W A=B$ consists of the following steps:

(1) Compute an SVD of $A$, yielding matrices $U, S$ and V:

if $n \geq k$, decompose $A$ such that $A=U S V^{r}$,

if $n<k$, decompose the transpose $A^{T}$ ouch that $A^{T}=V S U^{T}$,

where $U(n \times p)$ and $V(k \times p)$ contain the left and right singular vectors, respectively, and $V^{T}$ is the transpose of $V ; S$ is a dingonal $(p \times p)$ which contains $p$ non-zero aingular values $s_{1} \geq s_{2} \geq$ $\ldots \geq o_{p}>0$ and $p \leq \min (k, n)$

(2) Compute the mapping function $W=B V S^{-1} U^{T}$, where $S^{-1}=\operatorname{diag}\left(1 / s_{1}, 1 / s_{2}, \ldots, 1 / s_{p}\right)$.
3. Mapping arbitràry queries to canonical terms The LLSF mapping consists of the following steps:

(1) Given an arbitrary text (a "query"), first form a query vector, $\vec{x}$, in the source vector opace.

A query vector is similar to a column of matrix $A$, whose elements contain the numbers of times source words occur in the query. A query may also contain some words which are not in the source language; we ignore these words because no meaningful connections with them are provided by the mapping function. As an example, query severe stomach ulceration is converted into vector $\vec{x}=\left(\begin{array}{lllllll}0 & 0 & 0 & 0 & 0 & 1 & 1\end{array}\right)$.

(2) Transform the source vector $\vec{x}$ into $\vec{y}=W \vec{x}$ in the target apace.

In out example, $\vec{y}=W \vec{x}=\left(\begin{array}{llllll}0.375 & 0.5 & 0.5 & -0.25 & -0.25\end{array}\right.$ 0.375 ). Differing from text vectors in $A$ and term vectors in $B$, the elements (coefficients) of $\vec{y}$ are not limited to non-negative integers. These numbers show how the meaning of a query distributes over the words in the target language.

(3) Compare query-term similarity for all the term vectors and find the relevant terms.

In linear algebra, cosine-theta (or dot-product) is a common measure for obtaining vector similarity. It is also widely accepted by the information retrieval community using vector-based techniques because of the reasonable underlying intuition: it captures the similarity of texts by counting the similarity of individual words and then summarixing them. We use the cosine value to evaluate query-term similarity, defined as below:

Definition 2 . Let $\vec{y}=\left(y_{1}, y_{2}, \ldots, y_{m}\right)$ be the query vector in the target space and $\vec{v}=\left(v_{1}, v_{2}, \ldots, v_{m}\right)$ be a term vector in the target space,

$$
\begin{aligned}
& \operatorname{similarity}(\vec{y}, \vec{v})=\cos (\vec{y}, \vec{v}) \\
& =\frac{y_{1} v_{1}+y_{2} v_{2}+\ldots+y_{m} v_{m}}{\sqrt{v_{1}^{2}+y_{2}^{2}+\ldots+y_{m}^{2}} \sqrt{v_{1}^{2}+v_{2}^{2}+\ldots+v_{m}^{2}}}
\end{aligned}
$$

In order to find the closest match, we need to compare $\vec{y}$ with all the term vectors. We use $C$ to denote the matrix of these vectors distinct from matrix $B$ which represents the term collection in the training set. In general only a subset of terms are contained in a training set, so $C$ has more columns than the unique columns of $B$. Furthermore, $C$ could have more rows than $B$ becaube of the larger vocabulary. However, since only the words in $B$ have meaningful connections in the LLSF mapping function, we use the words in $B$ to form a reduced target language and trim $C$ into the same row as $B$. Words not in the reduced target language are ignored.

An exhaustive comparison of the query-term similarity 
values provides a ranked list of all the terms with respect to a query. A retrieval threshold can be chosen for drawing a line between relevant and irrelevant. Since relevance is often a relative concept, the choice of the threshold is left to the application or experiment.

A potential weakness of this method is that the term vectors in matrix $C$ are all surface-based (representing word occurrence frequency only) and are not affected by the training set or the mapping function. This weakness can be attenuated by a refined mapping method using a reverse mapping function $R$ which is an LLSF solution of the linear system $R B=A$. The refinement is described in a separate paper [8].

\section{The results}

\subsection{The primary test}

We tested our method with texts collected from patient records of Mayo Clinic. The patient records include diagnoses (DXs) written by physicians, operative reports written by surgeons, etc. The original texts need to be classified into canonical categories and about $1.5 \mathrm{mil}-$ lion patient records are coded by human experts each year. We arbitrarily chose the cardiovascular disease subset from the 1990 surgical records for our primary test. After human editing to separate these texts from irrelevant parts in the patient records and to clarify the one-to-one correspondence between $\mathrm{DX} s$ and canonical terms, we obtained a set of $6,913 \mathrm{DX} /$ term pairs. The target language consists of 376 canonical names of cardiovascular diseases as defined in the classification system ICD-8-CM [9]. A simple preprocessing was applied to remove punctuation and numbers, but no stemming or removal of non-discriminative words were used.

We split the 6,913 $\mathrm{DX}_{8}$ into two halves, called "oddhalf" and "even-half". The odd-half was used as the training set, the even-half was used as queries, and the expert-assigned canonical terms of the even-half were used to evaluate the effectiveness of the LLSF mapping. We used conventional measures in the evaluation: recall and precision, defined as

$$
\begin{aligned}
& \text { recall }=\frac{\text { terms retrieved and relevant }}{\text { total terms relevant }} \\
& \text { precision }=\frac{\text { terms retrieved and relevant }}{\text { total terms retrieved }} .
\end{aligned}
$$

For the query set of the even-half, we had a recall rate of $84 \%$ when the top choice only was counted and $96 \%$ recall among the top five choices. We also tested the odd-half, i.e. the training set itself, as querics and had a recall of $92 \%$ with the top choice and $99 \%$ with the top five. In our testing set, each text has one and only one relevant (or correct) canonical term, so the recall is always the same as the precision at the top choice.

Our experiments] system is implemented as a combination of $\mathrm{C}++$, Perl and UNIX shell programming.
For SVD, currently we use a matrix library in $\mathrm{C}++$ [10] which implements the same algorithm as in LINPACK[11]. A test with 3,457 pairs in the training set took about 4.45 hours on a SUN SPARCatation 2 to compute the mapping function $W$ and $R$. Since the computation of the mapping function is only needed once until the data collection is renewed, a real time response is not required. Term retrieval took $0.45 \mathrm{sec}$ or less per query and was satisfactory for practical needs. Two person-days of human editing were needed for preparing the testing set of the 6,913 DXs.

\subsection{The comparison}

For comparing our method with other approaches, we did additional tests with the same query set, the evenhalf $(3,456 \mathrm{DX} s)$, and matched it agajnst the same term set, the 376 ICD-8-CM disense categories.

For the test of a string matching method, we formed one matrix for all the 3,456 texts and the 376 terms, and used the cosine measure for computing the similarities. Only a $15 \%$ recall and precision rate was obtained at the top choice threshold.

For testing the effect of linguistic canonicalization, we employed a morphological parser developed by the Evans group at CMU [12] (and refined by our group by adding synonyms) which covers over 10,000 lexical variants. We used it as a preprocessor which converted lexical variants to word roots, expanded abbreviations to full spellings, recognized non-discriminative categories such as conjunctions and prepositions and removed them, and converted synonyms into canonical terms. Both the texts and the terms were parsed, and then the string matching as mentioned above was applied. The recall (and precision) rate was $17 \%$ (i.e. only $2 \%$ improvement), indicating that lexical canonicalization does not solve the crucial part of the problem; obviously, very little information was captured. Although synonyms were also used, they were a small collection and not especially favorable for the cardiovascular diseases.

For testing the effectiveness of statistical weighting, we ran the SMART system (version 10) developed at Cornell by Salton's group on our testing set. Two weighting schemes, one using term frequency and another using a combination of term frequency and "inverse document frequency", were tested with default parameters; $20 \%$ and $21 \%$ recall rates (top choice) were obtained, respectively. An interactive scheme using user feedback for improvement is also provided in SMART, but our tests did not include that option.

For further anaiysis we checked the vocabulary overlap between the query set and the term set. Only $20 \%$ of the source words were covered by the target words, which partly explains the unsatisfactory results of the above methods. Since they are all surface-based ap- 
Table 1.The ten summary of different methods

\begin{tabular}{|l|cc|}
\hline \multicolumn{1}{|c|}{ Method } & $\begin{array}{c}\text { rcall of } \\
\text { the top choice }\end{array}$ & $\begin{array}{c}\text { recall of } \\
\text { top five choices }\end{array}$ \\
\hline string matching & $15 \%$ & $42 \%$ \\
string matching enhenced by a morphological persing & $17 \%$ & $46 \%$ \\
SMART: rutirtical weighting uring IDF & $21 \%$ & $48 \%$ \\
LSSF: training wet = odd-hwlf & $84 \%$ & $96 \%$ \\
LLSF: training wet = odd-half, query set = odd-half & $92 \%$ & $99 \%$ \\
\hline
\end{tabular}

(1) The "even-half' (3,456 DXs) was urod as the query set for testing all the mothods atove, except the last one;

(2) the "odd-half" (3,457 DXs) was used as the training set in the LLSF' tests, which formed a source language including 945 distinct wonds and a turget langluge (reduced) including 376 urique cartonical terms and 224 distinct words; (3) the refined in

\begin{tabular}{|c|c|c|}
\hline 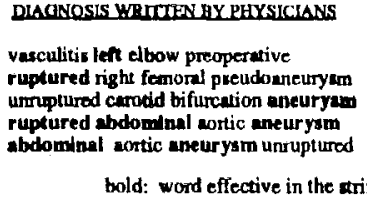 & 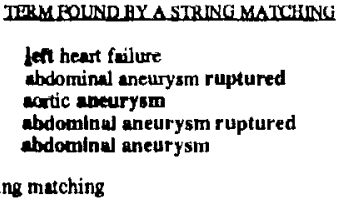 & $\begin{array}{l}\text { TERMEOLND BX THELUSE MAPPING } \\
\text { anteritis unipexified } \\
\text { anewrym of wtery of lower extremity } \\
\text { aneurym of artery of neck } \\
\text { abdominal aneurysm ruptured } \\
\text { abduminal aneurysm without mention } \\
\text { of rupture }\end{array}$ \\
\hline
\end{tabular}

Figure 2. Semple results of the DX-to-term mapping using the LLSF and a string matching method

proaches, only $20 \%$ of the query words were effectively used and roughly $80 \%$ of the information was ignored. These approaches share a common weakness in that they can not capture the implicit meaning of words (or only captured a little), and this seems to be a crucial problem.

The LLSF method, on the other hand, does not have such disadvantages. First, since the training set and the query set were from the same data collection, a much higher vocabulary coverage of $67 \%$ was obtained. Second, the $67 \%$ source words were further connected to their synonyms or related words by the LLSF mapping, according to the matches in the training set. Not only word co-occurrence, but also the contexts (sets of words) where the words have been used, were taken into account in the computation of weights; these connections were therefore context-sensitive. As a result, the $67 \%$ word coverage achieved an $84 \%$ recall and precision rate (top choice), outperforming the other methods by $63 \%$ or more. Table 1 summarizes these tests.

Figure 2 shows some sample results where each query is listed with the top choice by the LISF mapping and the top choice by the string matching. All the terms choaen by the LLSF mapping agreed with expert-assigned matches. It is evident that the LLSF mapping successfully captures the sernantic associations between the different surface expressions where as the string matching failed completely or missed important information.

\section{Discusaion}

\subsection{Irnpact to computational linguistics}

Recognizing word meanings or underlying concepts in natural language texts is a major focus in computational linguistics, especially in applied natural language processing such as information retrieval. Lexico-syntactic approaches have had limited achievement because lexical canonicalization and syntactic categorization can not capture much information about the implicit meaning of words and surface expressions. Knowledge-based approaches using semantic thesauri or networks, on the other hand, lead to the fundamental question about what should be put in a knowledge base. Is a general knowledge base for unrestricted subject areas realistic? If unlikely, then what should be chosen for a domain-specific or application-specific knowledge base? Is there a systematic way to avoid ad hoc decisions or the inconsistency that have often been involved in human development of semantic classes and the relationships between them? No clear answerg have been given for these quentions.

The LLSF method gives an effective solution for capturing semantic implications between surface expressions. The word-to-word connections between two languages capture synonyms and related terms with respect to the contexts given in the text/term pairs of the training set. Furthermore, by taking a training set from the same data collection as the queries the knowledge (semantics) is self-restricted, i.e. domain-specific, applicationspecific and user-group-specific. No symbolic representation of the knowledge is involved nor necessary, so subjective decisions by humans are avoided. As a re- 
sult, the $67-69 \%$ improvement over the string matching and the morphological parsing is evidence of our assertions.

\subsection{Difference from ather vector-based methods}

The use of vector/matrix representation, cosine measure and SVD makes our approach look similar to other vector-based methode, e.g. Salton's statistical weighting scheme and Deerwester's Latent Semantic Indexing (LSI) [13] which uses a word-document matrix and truncated SVD technique to adjust word weights in a document retrieval. However, there is a fundamental difference in that they focus on word weights based on counting word occurrence frequencies in a text collection, so only the words that appeared in queries and documents (terms in our context) have an affect on the retrieval. On the other hand, we focus on the weights of word-to-word connections between two languages, not weight of words; our computation is based on the information of human-assigned matches, the word cooccurrence and the contexts in the text/term pairs, not simply word occurrence frequencies. Our approach has an advantage in capturing synonyms or terms semantically related at various degrees and this makes a significant difference. As we discussed above, only $20 \%$ of query words were covered by the target words. So even if the statistical methods could find optimal weighto for these words, the majority of the information was still ignored, and as a result, the top choice recall and precision rate of SMART did not exceed $20 \%$ by much. Our tests with the LSI were mentioned in a separate paper [14]; the results were not better than SMART or the string matching method discussed above.

In short, besides the surface characteristics such as using matrix, cosine-theta and SVD, the LLSF mapping uses different information and solves the problem on a different scale.

\subsection{Potential applications}

We have demonstrated the success of the LLSF mapping in medical classification, but our method is not limited to this application. An attractive and practical application is automatic indexing of text databases and a retrieval using these indexing terms. As most existing text databases use human-assigned keywords for indexing documents, numerous amounts of document/term pairs can be easily collected and used as training sets. The obtained LLSF mapping functions then can be used for automatic document indexing with or without buman monitoring and refinement. Queries for retrieval can be mapped to the indexing terms using the same mapping functions and the rest of the task is simply a keyword-based search.

Another interesting potential is machine translation. Brown[15] proposed a statistical approach for machine translation which used word-to-word translation probability between two languages. They had about three million pairs of English-French sentences but the difficult problem was to breal the sentence-to-sentence association down to word-to-word. While they had a sophisticated algorithm to determine an alignment of word connections with maximum probability, it required estimation and re-estimation about possible alignments. Our LLSF mapping appears to have a great opportunity to diacover the optimal word-to-word translation probability, according to the English-French sentence pairs but without requiring any subjective estimations.

\subsection{Other aspects}

Several questions deserve a short discussion: is the word a good choice for the basis of the LLSF vector space? Is the LLSF the only choice or the best choice for a numerical mapping?

The word is not the only choice as the basis. We use it as a suitable starting point and for computational effciency. We also treat some special phrases such as $A C$ quired Immunodeficiency Syndrome as a single word, by putting hyphens between the words in a pre-formatting. An alternative choice to using words is to use noun phrases for invoking more syntactic constraints. While it may improve the precision of the mapping (how much is unclear), a combinatorial increase of the problem size is the trade-off.

Linear fit is a theoretical limitation of the LLSF mapping method. More powerful mapping functions are used in some neural networks[16]. However, the fact that the LLSF mapping is simple, fast to compute, and has well known mathematical properties makes it preferable at this stage of research. There are other numerical methods possible, e.g. using polynomial fit instead of linear fit, or using interpolation (going through points) instead of least squares fit, etc. The LLSF model demonstrated the power of numerical extraction of the knowledge from human-assigned mapping results, and finding the optimal solution among different fitting methods is a matter of implementstion and experimentation.

\section{Acknowledgement}

We would like to thank Tony Plate and Kent Bailey for fruitful discussions and Geoffrey Atkin for programming.

\section{References}

1. Blair DC, Maron ME. An evaluation of retrieval effectiveness of a full-text document-retrieval system. Communications of the $A C M$ 1985;28:289-299.

2. Chute CG, Yang $Y$, Evans DA. Latent semantic in- 
dexing of medical diagnoses using UMLS semantic structures. Proceedings of the 15th Annual Symposium on Computer Applications in Medical Care 1991;15:185189.

3. Evans DA, Handerson SK, Monarch IA, Pereiro J, Delon L, Hersh WR. Mapping vocabularies using "Latent Semantics." Technical Report No. CMU-LCL-91-1. Pittsburgh, PA: Carnegie Mellon University, 1991.

4. Salton G, Development in Automatic Text Retrieval, Science 1981:253:974-980.

5. Salton G, Yang CS, Wu CT. A theory of term importance in automatic text analysis. J Amer Soc Inf Sci 1975;26:33-44.

6. Lawson CL, and Hanson RJ. Solving Least Squares Problems. Englewood Cliffs, N.J.: Prentice-Hall, 1974.

7. Golub GH, Van Loan CE. Matrix Computations, and Edition. The Johns Hopkins University Press, 1989.

8. Yang Y, Chute CG. A Numerical Solution for Text information Retrieval and its Application in Patient Data Clasiification. Technical Report Series, No. 50, Section of Biostatistics, Mayo Clinic 1992.

9. International Classification of Diseases, 9th Revision, Clinical Modifications. Ann Arbor, MI: Commission on Professional and Hospital Activities, 1986.

10. M++ Class Library, User Guide, Release 9. Dyad Software Corporation; Bellevue, WA: 1991.

11. Dongarra JJ, Moler CB, Bunch JR, Stewart GW. LINPACK Users' Guide. Philadelphia, PA: SIAM, 1979.

12. Evans DA, Hersh WR, Monarch IA, Lefferts RG, Handerson SK. Automatic indexing of abstracts via natural-language processing using a simple thesaurus. Medical Decision Making 1991;11/4 Suppl;108-115.

13. Deerwester S., Dumais ST, Furnas GW, Landauer TK, Harshman R. Indexing by Latent Semantic Analysis. J Amer Soc Inf Sci 1990;41(6):391-407.

14. Chute CG, Yang Y. An Evaluation of Concept Based Latent Semantic Indexing for Clinical Information Retrieval. Proceedings of the 16th Annual Symposium on Computer Applications in Medical Care 1991; ted.

15. Brown PG, Cocke J, Pietra SD, Pietra VJD, Jelinek F, Lafferty JD, Mercer RL, Roossin PS. A Statistical Approach to Machine Translation. Computational Lingeistics, 1980;16(2): 79-85.

16. Rumelhart DE, McClelland JL and the PDP Research Group. Parallel Distributed Processing: Explorations in the Microstructure of Cognition. Cambridge, Mass.: MIT Press, 1986. 